\title{
Reflection and refraction of an Airy beam at a dielectric interface
}

\author{
Ioannis D. Chremmos* and Nikolaos K. Efremidis \\ Department of Applied Mathematics, University of Crete, Heraklion 71409, Greece \\ ${ }^{*}$ Corresponding author: jochremm@central.ntua.gr
}

Received January 11, 2012; accepted February 23, 2012;

posted February 29, 2012 (Doc. ID 161375); published May 11, 2012

\begin{abstract}
Reflection and refraction of a finite-power Airy beam at the interface between two dielectric media are investigated analytically and numerically. The formulation takes into account the paraxial nature of the optical beams to derive convenient field evolution equations in coordinate frames moving along Snell's refraction and reflection axes. Through numerical simulations, the self-accelerating dynamics of the Airy-like refracted and reflected beams are observed. Of special interest are the cases of critical incidence at Brewster and total-internal-reflection (TIR) angles. In the former case, we find that the reflected beam achieves self-healing, despite the severe suppression of a part of its spectrum, while, in the latter case, the beam remains nearly unaffected except for the GoosHänchen shift. The self-accelerating quality persists even if the beam is trapped by multiple TIRs inside a dielectric film. The grazing incidence of an Airy beam at the interface between two media with close refractive indices is also investigated, revealing that the interface can act as a filter depending on the beam scale and tilt. We finally consider reverse refraction and perfect imaging of an Airy beam into a left-handed medium. (c) 2012 Optical Society of America
\end{abstract}

OCIS codes: $\quad 240.0240,290.0290,120.5700,120.5710,050.1940$.

\section{INTRODUCTION}

The optics of Airy light beams is currently attracting increasing research interest. The Airy wave packet was first conceived in 1979 in the context of quantum mechanics as a nonspreading solution to the potential-free Schrödinger's equation [1]. Its salient property is the ability to freely accelerate in the absence of external forces. However, despite the striking similarity of the free-particle Schrödinger's equation ( $\Psi_{t}=i \hbar \Psi_{x x} / 2 m, m$ being the mass) with the paraxial approximation of light diffraction $\left(u_{z}=i u_{x x} / 2 k_{0}, k_{0}\right.$ being the wavenumber), it was not until 2007 that the feasibility of optical Airy wave packets, i.e., light beams with an Airy wavefront, was conceived [2]. These new light waves have two remarkable properties: diffraction-free propagation and transverse self-acceleration. As first predicted theoretically and later observed experimentally [3], the self-accelerating property is manifested by a parabolic, ballistic-like trajectory that the beam's local intensity features follow in space, giving the impression of a projectile moving under the influence of gravity [4]. This peculiar behavior of light bending in the absence of refractive index gradients is due to the inherent chirped phase modulation of the Airy function, which causes the constituent beam rays to form a parabolic caustic in space [5].

As happens with other known diffraction-free beams, such as Bessel [6], Mathieu [7]], or parabolic [8] beams, an ideal Airy beam carries infinite power and hence is not physically realizable. Hopefully, it has been shown that exponentially modulated (finite-power) Airy beams maintain the remarkable features of ideal Airy beams over several diffraction lengths before they are significantly distorted, thus lending themselves to practical use [2,3]. It should be emphasized that, although the self-bending behavior of finite-power Airy beams may provoke one's notion of light traveling along straight paths, a careful analysis reveals that the beam's centroid still follows a straight path. Hence Ehrenfest's momentum theorem is by no means violated [9].

Another remarkable feature of Airy beams is their robustness against scattering or turbulent environments as well as their ability to self-heal and reproduce their wavefront even after they are severely perturbed or obstructed $[\underline{10}, 11]$. Other works have also shown the persistence of their features inside self-defocusing nonlinear media [12]. All these unique properties have already been utilized to open a number of application fields, such as beam trajectory control [13], optical micromanipulation $[14,15,16]$, vacuum electron acceleration [17], curved plasma filaments [18], Airy plasmon-polaritons $[19,20,21]$, and abruptly autofocusing waves [22].

Additionally, the analogy between diffraction of optical beams in space and dispersion of optical pulses in time has also motivated the realization of Airy wave packets in the time domain [2]. The resulting Airy pulses can propagate with acceleration and minimum shape distortion over several dispersion lengths in media with quadratic or cubic dispersion [23]. These unique waveforms have so far found application for generating dispersion- and diffraction-resisting optical bullets [24], optical solitons [25], and supercontinua [26].

In the present work, phenomena associated with the refraction and reflection of a finite-power Airy beam at a dielectric interface are investigated analytically and numerically, for the first time to our knowledge. The problem is of interest in optical settings where Airy beams are targeted into dielectric media, exit the medium in which they have been generated, or propagate through layers, such as biological tissues. For the sake of simplicity and in order to focus on the essentials, we here restrict ourselves to one-dimensional (1D) Airy beams, hence to two-dimensional (2D) scattering configurations. 
The extension to 2D Airy beams and three-dimensional (3D) scattering is immediate. The analytical formulation takes into account the paraxial nature of an Airy beam to derive the evolution of the field amplitude in convenient coordinate frames that move along Snell's refraction and reflection beam axes.

Through numerical simulations, we find that, away from critical angles, the reflected and refracted beams have a very Airy-like amplitude profile, thus retaining the self-accelerating and diffraction-resisting qualities of the incident wave. The acceleration and diffraction rate of the transmitted beam is however reduced or increased if the second medium is optically denser or thinner, respectively. Of special interest are the cases of critical incidence at Brewster and total-internalreflection (TIR) angles. In the Brewster case, we find that the $p$-polarized reflected beam achieves self-healing and reconstructing its Airy profile, despite the severe filtering of its spectrum, which is a unique feature. In the TIR case, the beam remains nearly unaffected, experiencing only a slight lateral shift due to the Goos-Hänchen effect. Subsequently, and assuming that the beam undergoes multiple TIRs inside a thin dielectric slab, we also find that the self-accelerating character persists, however at the cost of increased diffraction. Another interesting case is that of two media whose refractive indices are very close. In this case the TIR phenomenon occurs at grazing angles and a paraxial propagation formulation must be adopted. It is found that the interface can act as a selective filter depending on the transverse scale and initial tilt of the impinging Airy beam. Moreover, in TIR the interference between the incident and reflected beams creates an interesting interference pattern similar to that observed with abrupt autofocusing waves. We finally consider the interface to a left-handed medium (LHM). In this case the transmitted beam refracts along the reversed Snell direction and creates a perfect image of itself at a certain depth inside the LHM, while still accelerating toward the same direction with the incident beam.

\section{ANALYSIS}

Consider the 2D problem of Fig. 1, where a monochromatic optical beam with time dependence $\exp (-i \omega t)$ impinges at an angle $\theta_{1}$ on the plane interface between two dielectric halfspaces. The two media are characterized by dielectric constants $\varepsilon_{1}, \varepsilon_{2}$, magnetic permeabilities $\mu_{1}, \mu_{2}$, and wavenumbers $k_{1}=\omega\left(\varepsilon_{1} \mu_{1}\right)^{1 / 2}, k_{2}=\omega\left(\varepsilon_{2} \mu_{2}\right)^{1 / 2}$. Let also $z=-h$ be the depth

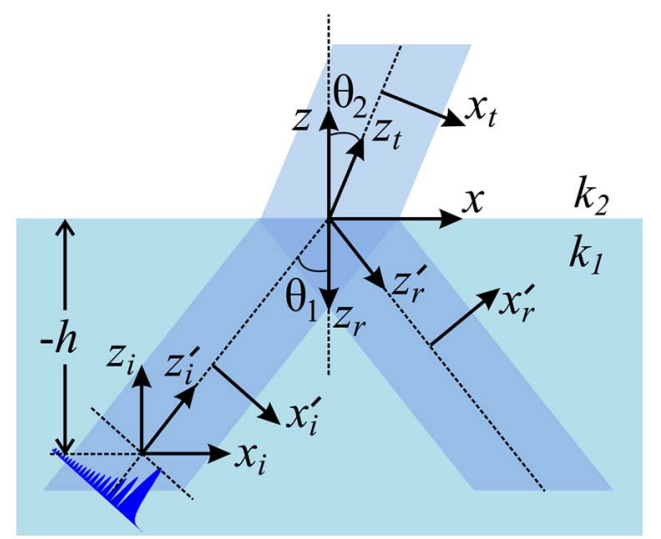

Fig. 1. (Color online) Problem geometry and the different coordinate systems used. Axis $x_{r} \equiv x$ is not shown. inside medium 1 , at which the beam axis intersects its input plane. For convenience in expressing the incident, reflected, and refracted beams, we also introduce the following coordinate systems:

- $\left(x_{i}, z_{i}\right)=(x+s, z+h), s=h \tan \theta_{1}$. This system follows from a translation of the "interface" system $(x, z)$ to the point $(s, h)$ where the beam axis intersects its input plane.

- $\left(x_{i}^{\prime}, z_{i}^{\prime}\right)=\left(x_{i} \cos \theta_{1}-z_{i} \sin \theta_{1}, x_{i} \sin \theta_{1}+z_{i} \cos \theta_{1}\right)$. This system follows from a clockwise rotation of system $\left(x_{i}, z_{i}\right)$ by $\theta_{1}$ and is aligned with the incident beam axis.

- $\left(x_{r}, z_{r}\right)=(x,-z)$. This is system $(x, z)$ with reversed $z$ axis in order to follow the dynamics of the reflected beam toward positive $z_{r}$.

- $\quad\left(x_{r}^{\prime}, z_{r}^{\prime}\right)=\left(x_{r} \cos \theta_{1}-z_{r} \sin \theta_{1}, x_{r} \sin \theta_{1}+z_{r} \cos \theta_{1}\right)$ This system follows from an anticlockwise rotation of system $\left(x_{r}, z_{r}\right)$ by $\theta_{1}$ and is aligned with the reflected beam axis.

- $\left(x_{t}, z_{t}\right)=\left(x \cos \theta_{2}-z \sin \theta_{2}, x \sin \theta_{2}+z \cos \theta_{2}\right)$.

This system follows from a clockwise rotation of system $(x, z)$ by $\theta_{2}$, i.e., Snell's angle of refraction, and is aligned with the refracted beam axis. This is helpful only when $\theta_{2}$ exists. In other cases, the interface system is used.

Now let $\psi_{i}, \psi_{r}, \psi_{t}$ be, respectively, the incident, reflected, and refracted field amplitudes, representing the $y$-directed electric or magnetic field, in case of a $s$ - (TE) or $p$-(TM) polarized wave, respectively. To solve the scattering problem, one needs to define the input field at $z=-h$, or equivalently $z_{i}=0$. In general, this can be expressed as an arbitrary function $f\left(x_{i}\right)$. For a paraxial beam wave, however, whose axis is at an angle $\theta_{1}$ with the interface, it is convenient to write

$$
\psi_{i}\left(x_{i}, z_{i}=0\right)=u_{0}\left(x_{i}\right) \exp \left(i \beta_{0} x_{i}\right)
$$

where $u_{0}\left(x_{i}\right)$ is a slowly varying, with respect to the wavelength $\left(\lambda_{1}=2 \pi / k_{1}\right)$, envelope and $\beta_{0}=k_{1} \sin \theta_{1}$ can be viewed as the projection of the average (or central) beam wavenumber on the $x$ axis.

To determine the envelope function, one notices that, in coordinates $\left(x_{i}^{\prime}, z_{i}^{\prime}\right)$ the amplitude of the paraxial beam is written $\psi_{i}\left(x_{i}^{\prime}, z_{i}^{\prime}\right)=f_{0}\left(x_{i}^{\prime}, z_{i}^{\prime}\right) \exp \left(i k_{1} z_{i}^{\prime}\right)$. Then on plane $z_{i}=0$, one substitutes $x_{i}^{\prime}=x_{i} \cos \theta, z_{i}^{\prime}=x_{i} \sin \theta_{1}$ to obtain $u_{0}\left(x_{i}\right)=$ $f_{0}\left(x_{i} \cos \theta_{1}, x_{i} \sin \theta_{1}\right)$. However, thanks to paraxiality, the variations of $f_{0}$ with respect to $z_{i}^{\prime}$ occur on a much larger length scale than variations with respect to $x_{i}^{\prime}$. This allows one to approximate

$$
u_{0}\left(x_{i}\right) \cong f_{0}\left(x_{i} \cos \theta_{1}, 0\right),
$$

which is valid when $\theta_{1}$ is not too close to $90^{\circ}$. Specifically, Eq. (2) can be used with confidence when $k_{1} x_{0} \cot \theta_{1} \gg 1$, $x_{0}$ being the scale of variations of $f_{0}\left(x_{i}^{\prime}, 0\right)$.

Through a Fourier transform (FT) in coordinates $(x, z)$, the incident field is decomposed into plane waves. Subsequently, each reflected or transmitted plane wave component is weighted according to the corresponding Fresnel coefficients. Taking the inverse FT, the incident, reflected, and transmitted waves read

$$
\psi_{i}(x, z)=\frac{1}{2 \pi} \int_{-\infty}^{+\infty} \Psi(k) \exp \left(i k x+i q_{1}(z+h)\right) \mathrm{d} k
$$




$$
\begin{gathered}
\psi_{r}(x, z)=\frac{1}{2 \pi} \int_{-\infty}^{+\infty} \Psi(k) R(k) \exp \left(i k x-i q_{1}(z-h)\right) \mathrm{d} k, \\
\psi_{t}(x, z)=\frac{1}{2 \pi} \int_{-\infty}^{+\infty} \Psi(k) T(k) \exp \left(i k x+i q_{2} z+i q_{1} h\right) \mathrm{d} k,
\end{gathered}
$$

where $\Psi(k)$ is the FT of the input wave and $q_{1}=\left(k_{1}^{2}-k^{2}\right)^{1 / 2}$, $q_{2}=\left(k_{2}^{2}-k^{2}\right)^{1 / 2}$ are the $z$ wavenumbers of the Fourier components in the two media. Radiation conditions require that the square root signs are chosen so that $\operatorname{Re}\left(q_{1,2}\right)>0$, $\operatorname{Im}\left(q_{1,2}\right)>0$. By applying boundary conditions at the interface, i.e., continuity of the tangential electric and magnetic field components, the Fresnel reflection and refraction coefficients are expressed by

$$
R(k)=\frac{\varepsilon_{2} q_{1}-\varepsilon_{1} q_{2}}{\varepsilon_{2} q_{1}+\varepsilon_{1} q_{2}}, \quad T(k)=\frac{2 \varepsilon_{2} q_{1}}{\varepsilon_{2} q_{1}+\varepsilon_{1} q_{2}}
$$

for $p$-polarized waves. For $s$-polarized waves, the same expressions hold but with permittivities $\varepsilon_{1,2}$ replaced by permeabilities $\mu_{1,2}$. Now from Eqs. (1), (2) and $x_{i}=x+s$, the FT of the input wave is given by

$$
\Psi(k)=\int_{-\infty}^{+\infty} \psi_{i}(x,-h) \exp (-i k x) \mathrm{d} k=\frac{\exp (i k s)}{\cos \theta_{1}} U_{0}\left(\frac{k-\beta_{0}}{\cos \theta_{1}}\right)
$$

where $U_{0}(k)$ is the FT of $f_{0}\left(x_{i}^{\prime}, 0\right)$. Subsequently, Eq. (5) is substituted into Eqs. (3) and the integration variable is changed to $\xi=k-\beta_{0}$. For a paraxial incident beam, the length scale $x_{0}$ is much larger than the optical wavelength; hence, the width of the spectrum $\Psi(\xi)$ is sufficiently narrow to motivate the Taylor expansion

$$
q_{1}(\xi)=\sqrt{k_{1}^{2}-\left(\beta_{0}+\xi\right)^{2}}=q_{10}-\frac{\beta_{0}}{q_{10}} \xi-\frac{k_{1}^{2}}{2 q_{10}^{3}} \xi^{2}-\frac{\beta_{0} k_{1}^{2}}{2 q_{10}^{5}} \xi^{3}-\ldots
$$

where $q_{10}=\left(k_{1}^{2}-\beta_{0}^{2}\right)^{1 / 2}=k_{1} \cos \theta_{1}$. Now if $\beta_{0} \xi \ll q_{10}^{2}$ (which is equivalent to the previously mentioned condition $k_{1} x_{0} \cot \theta_{1} \gg 1$, since $\xi$ is of the order $x_{0}^{-1} \cos \theta_{1}$ ), one may keep only terms up to second order and obtain for the reflected beam

$$
\begin{aligned}
\psi_{r}\left(x_{r}, z_{r}\right) \cong & u_{r}\left(x_{r}-z_{r} \tan \theta_{1}, z_{r}\right) \exp \left(i \beta_{0}\left(x_{r}+s\right)\right. \\
& \left.+i q_{10}\left(h+z_{r}\right)\right),
\end{aligned}
$$

where the envelope function is given by the Fourier integral

$$
\begin{aligned}
u_{r}\left(\chi_{r}, z_{r}\right)= & \frac{1}{2 \pi \cos \theta_{1}} \int_{-\infty}^{+\infty} U_{0}\left(\frac{\xi}{\cos \theta_{1}}\right) R\left(\beta_{0}+\xi\right) \\
& \times \exp \left[i \xi \chi_{r}-i \frac{\xi^{2} k_{1}^{2}\left(z_{r}+h\right)}{2 q_{10}^{3}}\right] \mathrm{d} \xi .
\end{aligned}
$$

In the latter equation, $\chi_{r}=x_{r}-z_{r} \tan \theta_{1}$ is the reduced $x$ coordinate centered at the line $x_{r}=z_{r} \tan \theta_{1}$, namely the reflected beam axis predicted by Snell's law and it is the counterpart of the retarded time frame moving at the group velocity of an optical pulse.
The interpretation of Eqs. () and () is obvious. The reflected beam amplitude is concentrated around the axis predicted by Snell's law, and its spectrum has been modulated by the Fresnel reflection coefficient. With increasing $z_{r}$, the beam experiences diffraction, due to the quadratic phase $\left(\xi^{2}\right)$ acquired by its spectrum. The quadratic phase term is proportional to $z_{r}+h$, thus accounting for the diffraction of the incident beam along the entire path from the input to the observation plane. In addition, as shown by the exponential factor in Eq. (7), the beam acquires a phase equal to $k_{1}\left[\left(x_{r}+s\right)^{2}+\left(z_{r}+h\right)^{2}\right]^{1 / 2}$, i.e., $k_{1}$ times the path propagated along the beam axis from the input to the observation plane.

Turning to the wave transmitted through the interface, one needs to consider two regimes. When the angle of incidence is below the critical TIR angle $\theta_{c}=\sin ^{-1}\left(k_{2} / k_{1}\right)$, then there is a well-defined refracted beam at the angle $\theta_{2}$ predicted by Snell's law: $k_{1} \sin \theta_{1}=k_{2} \sin \theta_{2}$. If additionally, $|\xi| \ll q_{20} \cot \theta_{2}$, where $q_{20}=\left(k_{2}^{2}-\beta_{0}^{2}\right)^{1 / 2}=k_{2} \cos \theta_{2}$ one approximates $q_{2}(\xi)$ similar to $q_{1}(\xi)$ in Eq. (ㅁ) and substitutes in Eq. (3.3) to obtain

$$
\psi_{t}(x, z) \cong u_{t}\left(x-z \tan \theta_{2}, z\right) \exp \left[i \beta_{0}(x+s)+i q_{20} z+i q_{10} h\right]
$$

with

$$
\begin{aligned}
u_{t}\left(\chi_{t}, z\right)= & \frac{1}{2 \pi \cos \theta_{1}} \int_{-\infty}^{+\infty} U_{0}\left(\frac{\xi}{\cos \theta_{1}}\right) T\left(\beta_{0}+\xi\right) \\
& \times \exp \left[i \xi \chi_{t}-i \frac{\xi^{2}}{2}\left(\frac{k_{2}^{2} z}{q_{20}^{3}}+\frac{k_{1}^{2} h}{q_{10}^{3}}\right)\right] \mathrm{d} \xi,
\end{aligned}
$$

$\chi_{t}=x-z \tan \theta_{2}$ being the reduced $x$ coordinate. The interpretation of Eqs. (9) and (10) is similar to that for the reflected beam. Note in Eq. (10) that the quadratic (diffracting) phase is the sum of two components because the wave has propagated through two different media. If the second medium is left handed, i.e., of a negative refractive index, then the second term is of the opposite sign, thus tending to eliminate the diffraction accumulated in the first medium. At a certain depth inside the medium, the two terms cancel exactly and perfect imaging is accomplished. An example will be investigated in Section 3 .

In the TIR regime on the other hand, there does not exist a refraction angle $\theta_{2}$, and $q_{2}(\xi)$ becomes imaginary in a part of the spectrum. Then, by approximating only wavenumber $q_{1}(\xi)$ according to Eq. (ㅁ), we obtain

$$
\psi_{t}(x, z) \cong u_{t}(x, z) \exp \left(i \beta_{0}(x+s)+i q_{10} h\right),
$$

where

$$
\begin{aligned}
u_{t}(x, z)= & \frac{1}{2 \pi \cos \theta_{1}} \int_{-\infty}^{+\infty} U_{0}\left(\frac{\xi}{\cos \theta_{1}}\right) T\left(\beta_{0}+\xi\right) \\
& \times \exp \left(i \xi x+i q_{2} z-i \frac{k_{1}^{2} h}{2 q_{10}^{3}} \xi^{2}\right) \mathrm{d} \xi .
\end{aligned}
$$

It is interesting to notice that Eqs. (8) and (10) are the solution to the paraxial equation of light diffraction for the reflected and refracted beams in coordinates $\left(\chi_{r}, z_{r}\right),\left(\chi_{t}, z\right)$, respectively, under the appropriate initial conditions. For example, for the reflected beam, the paraxial equation 


$$
\frac{\partial u_{t}}{\partial z}=i \frac{k_{2}^{2}}{2 q_{20}^{3}} \frac{\partial^{2} u_{t}}{\partial \chi_{t}^{2}}
$$

is implied, subject to the initial condition $u_{t}\left(\chi_{t}, 0\right)$, obtained from Eq. (10). In the TIR regime, such an approximation is not valid and Eq. (12) implies the complete Helmholtz equation.

We close this section by investigating the case of grazing incidence $\left(\theta_{1}\right.$ close to $\left.90^{\circ}\right)$ of an Airy beam at the interface. This is interesting when the refractive indices of the two media are very close $\left(0<n_{1}-n_{2} \ll n_{2}\right)$ and, consequently, the critical TIR angle is also close to $90^{\circ}$. In this case, the FT approach of Eqs. (3) is inefficient because both reflected and transmitted rays remain almost parallel to the interface, thus implying the applicability of the paraxial approximation to the Helmholtz equation. The total field on both sides of the interface is then expressed as $u(x, z) \exp \left(i k_{1} x\right)$, where the slowly varying in $x$ envelope $u$ satisfies the parabolic differential equation

$$
i 2 k_{1} \frac{\partial u}{\partial x}+V(z) u+\frac{\partial^{2} u}{\partial z^{2}}=0, \quad x>0
$$

with the index discontinuity creating the "potential" $V(z)=$ $\left(k_{2}^{2}-k_{1}^{2}\right) H(z)$, with $H$ being the Heaviside function. The input condition is defined on plane $x=0$ as $u(0, z)$ and, for a beam impinging from medium 1 , must be confined in half-space $z<0$.

\section{NUMERICAL RESULTS}

Let us now apply the above to numerically investigate the reflection and refraction dynamics of Airy beams. In the following simulations, the two media are taken to be nonmagnetic $\left(\mu_{1}=\mu_{2}=\mu_{0}\right)$ with refractive indices $n_{1}=1.5, n_{2}=1.0$, leading to a critical TIR angle $\theta_{c}=41.81^{\circ}$. The vacuum wavelength is fixed at $\lambda=500 \mathrm{~nm}$. For a finite-power Airy beam the transverse input amplitude is

$$
f_{0}\left(x_{i}^{\prime}, z_{i}^{\prime}=0\right)=A i\left( \pm \frac{x_{i}^{\prime}}{x_{0}}\right) \exp \left( \pm a \frac{x_{i}^{\prime}}{x_{0}}\right)
$$

where $a$ is the truncation (or, as commonly referred to, apodization) parameter and the \pm sign determines the direction toward which the Airy lobes develop and, consequently, the direction of acceleration. The FT of this wave function is known in closed form to be [2]

$$
\begin{aligned}
U_{0}(k) & =\int_{-\infty}^{+\infty} f_{0}\left(x_{i}^{\prime}, z_{i}^{\prime}=0\right) \exp \left(-i k x_{i}^{\prime}\right) \mathrm{d} x_{i}^{\prime} \\
& =x_{0} \exp \left[\frac{i}{3}\left( \pm k x_{0}+i a\right)^{3}\right] .
\end{aligned}
$$

As a first example, consider a $p$-polarized Airy beam, with $x_{0}=20 \mu \mathrm{m}, a=0.08$, and lobes developing toward negative $x$ [positive sign in Eq. (14)] impinging on the interface at an angle $\theta_{1}=30^{\circ}$ The diffraction length for this beam in medium 1 is $L_{d 1}=k_{1} x_{0}^{2} \cong 7.5 \mathrm{~mm}$, and we also let $h=L_{d 1} \cos \theta_{1} \cong$ $6.5 \mathrm{~mm}$ so that the beam is allowed to propagate for one diffraction length before it hits the interface. The reflected and refracted beam amplitudes have been computed using Eqs. (8) and (10) and are shown in Figs. 2(a) and 2(b), respectively.
As explained in Section 2 , reduced coordinates $\left(\chi_{r}, z_{r}\right),\left(\chi_{t}, z\right)$ are used that follow the beam axes, which are at angles $\theta_{1}=30^{\circ}$ for the reflected and $\theta_{2}=48.6^{\circ}$ for the refracted beam. Both beams are Airy-like and accelerate toward the same direction with the incident beam, as a result of the cubic phase modulation of the incident beam spectrum [Eq. (15)]. The equations of the reflected and refracted caustics can be determined by a stationary-phase approach to the Fourier integrals of Eqs. (8) and (10). Differentiating the phase of the integrand and including the cubic phase of the Airy function FT [Eq. (15)], one obtains a quadratic equation. The caustics follow from setting the discriminant equal to zero, and the equations read

$$
\begin{aligned}
& \chi_{r}= \pm \frac{\left(h+z_{r}\right)^{2}}{4 k_{1}^{2} x_{0}^{3} \cos ^{3} \theta_{1}}, \\
& \chi_{t}= \pm \frac{1}{4 k_{1}^{2} x_{0}^{3} \cos ^{3} \theta_{1}}\left(h+\frac{k_{1} \cos ^{3} \theta_{1}}{k_{2} \cos ^{3} \theta_{2}} z\right)^{2}
\end{aligned}
$$

for the reflected and refracted caustic, respectively, while \pm corresponds to the sign of Eq. (14). Hence, both beams follow a parabolic trajectory, however, with a different acceleration rate and initial launch angles. It can be shown that the launch angles, i.e., $\tan ^{-1}\left(d \chi_{r} / d z_{r}\right)_{z_{r}=0}$ and $\tan ^{-1}\left(d \chi_{t} / d z\right)_{z=0}$, at which the caustics originate from the interface and the angle at which the incident parabolic caustic hits the interface, are consistent with Snell's law. The split of the incident caustic to the reflected and refracted caustic occurs at $x= \pm h^{2}$ / $\left(4 k_{1}^{2} x_{0}^{3} \cos ^{3} \theta_{1}\right)$. The caustics of Eq. ( $\left.\underline{16}\right)$ have been superposed in Fig. 2 .

Now consider the same $p$-polarized Airy beam impinging at Brewster's angle $\theta_{B}=\tan ^{-1}\left(n_{2} / n_{1}\right)=33.69^{\circ}$. Then the Fresnel reflection coefficient in Eq. ()ㅜ has a zero for $\xi=0\left(R\left(\beta_{0}\right)=0\right)$, and, as a result, the spectrum of the reflected beam is severely disturbed and attenuated. The distortion of the beam shape is evident in Fig. 3(a), where the suppression of the central $(\xi=0)$ part of the spectrum is
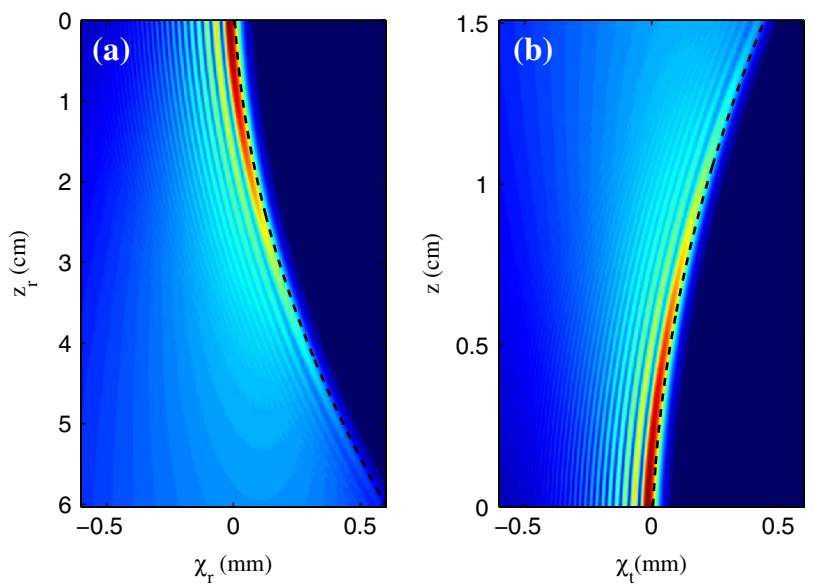

Fig. 2. (Color online) (a) Reflected and (b) refracted beam amplitude for a $p$-polarized finite-power Airy beam $\left(x_{0}=20 \mu \mathrm{m}, a=0.08\right)$ impinging at an angle $\theta_{1}=30^{\circ}$ on a glass-air interface $\left(n_{1}=1.5\right.$, $\left.n_{2}=1.0\right)$. The incident beam has propagated for one diffraction length before it reaches the interface. According to the defined coordinate systems, in (a) $z_{r}$ is the depth inside the dielectric and $\chi_{r}$ measures the horizontal position with respect to Snell's reflection axis; in (b) $z$ is the height in the air and $\chi_{t}$ measures horizontal position with respect to Snell's refraction axis $\left(\theta_{2}=48.6^{\circ}\right)$. Dotted curves are the caustics of Eq. (16). 
manifested by a dark lobe around $\chi_{r}=0$ (beam axis) and by the strongly distorted initial amplitude profile (at $z_{r}=0$ ), shown in Fig. 3(b). However, a part of the Airy spectrum survives and manages to reconstruct the caustic as the beam propagates and, as seen from Fig. 3(b), the features of the Airy profile have been recovered by depth $z_{r}=3 \mathrm{~cm}$.

For an Airy beam whose lobes develop to negative $x$, it is the positive spatial frequencies $(\xi>0)$ that correspond to the rays forming the parabolic caustic [5]. Hence, if the angle of incidence $\theta_{1}$ is slightly increased beyond the Brewster angle so that the zero of the reflection coefficient $R\left(\xi+\beta_{0}\right)$ occurs at some negative $\xi$, then the reflected caustic will be essentially unaffected. This is the case of Fig. 4(a), where $\theta_{1}=$ $\theta_{B}+0.25^{\circ}$. Clearly, the caustic is unaffected, while the dark lobe manifests Brewster's suppression of a negative spatial frequency. On the other hand, if $\theta_{1}$ is slightly decreased below the Brewster angle, then a positive spatial frequency window is suppressed and the caustic is disturbed deeper inside medium 1, rather than at the interface. This case is shown in Fig. 4(b) for $\theta_{1}=\theta_{B}-0.25^{\circ}$ The reflected beam is now significantly distorted with some power diffracted toward negative $\chi_{r}$. As a result of the missing spatial frequencies, the main lobe near the caustic is suppressed around $z_{r}=2 \mathrm{~cm}$ with the corresponding missing ray manifesting itself as a dark lobe. Self-healing occurs at $z_{r}=4 \mathrm{~cm}$, beyond which a clear main Airy lobe is formed and persists.

Note that the total reflected power ratio at Brewster incidence is very low. Using Parseval's theorem and Eq. (ㅁ), the ratio of reflected power is

$$
P_{r}=\frac{\int_{-\infty}^{+\infty}\left|U_{0}\left(\xi / \cos \theta_{1}\right) R\left(\beta_{0}+\xi\right)\right|^{2} \mathrm{~d} \xi}{\int_{-\infty}^{+\infty}\left|U_{0}\left(\xi / \cos \theta_{1}\right)\right|^{2} \mathrm{~d} \xi},
$$

and it is around $4 \times 10^{-5}$ for the case of Fig. 3. For lossless dielectrics, the transmitted power ratio is found by energy conservation $T_{r}=1-P_{r}$.

Now let us consider the TIR case. In Figs. 5(a) and 5(b), the reflected and transmitted wave amplitudes are shown when the Airy beam of the previous examples impinges at $\theta_{1}=\theta_{c}=41.81^{\circ}$. Because of TIR, there is no refracted beam
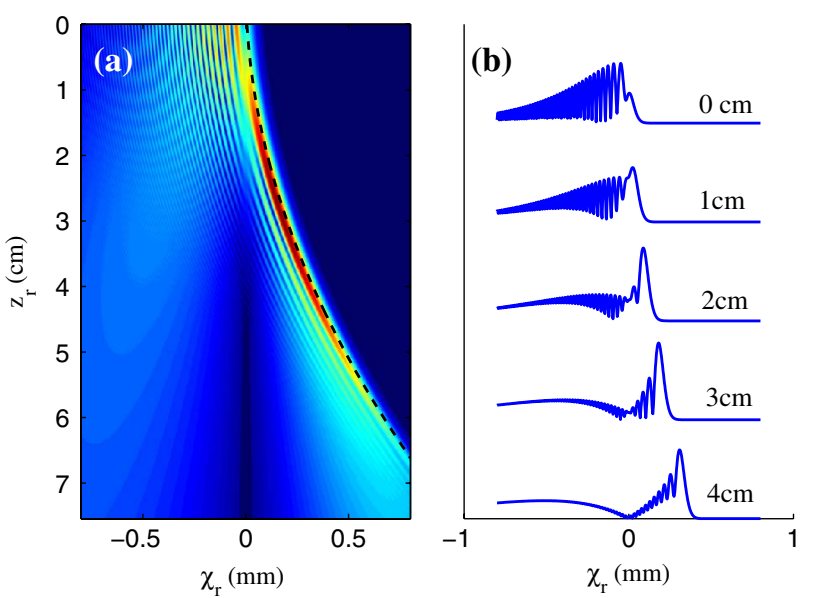

Fig. 3. (Color online) (a) Reflected beam amplitude for the Airy beam of Fig. 2, impinging at Brewster's angle $\theta_{1}=33.69^{\circ}$ on the same glass-air interface. The dotted curve is the caustic. (b) Beam amplitude (a.u.) versus position $\chi_{r}$ from Snell's reflection axis at different indicated depths inside the glass.
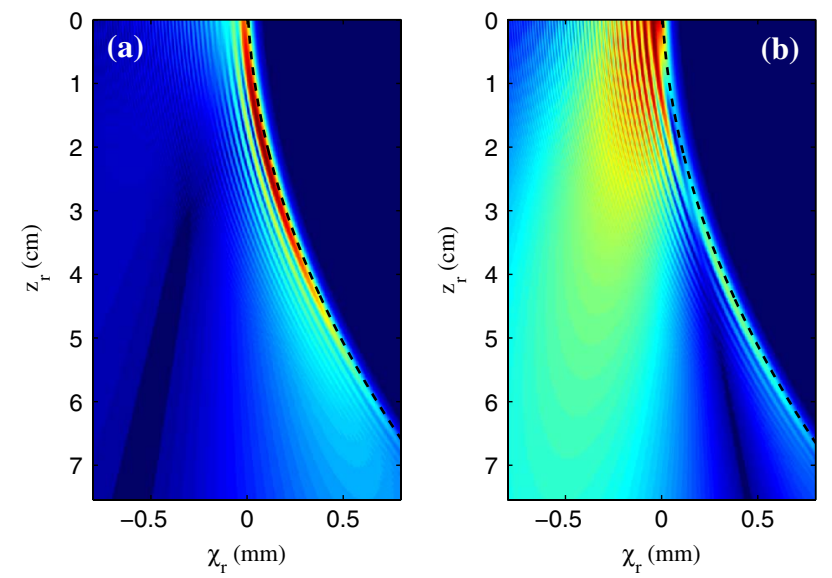

Fig. 4. (Color online) Reflected beam amplitudes for the Airy beam of Fig. 2, impinging at angle (a) $\theta_{1}=\theta_{B}+0.25^{\circ}=33.94^{\circ}$ and (b) $\theta_{1}=\theta_{B}-0.25^{\circ}=33.44^{\circ}$ on the same dielectric-air interface. The dotted curves are the caustics.

but only an evanescent, Airy-like field distribution that dies away a few micrometers away from the interface. As explained in Section 2, the interface system $(x, z)$ is used in Fig. 5(b). In reflection, an Airy-like beam is formed, with a clear accelerating dynamic with respect to Snell's reflection axis $\left(\chi_{r}=0\right)$. In Fig. 5(c) the Gaussian amplitude of the incident beam's spectrum $\left[U_{0}\left(\xi / \cos \theta_{1}\right)\right]$ is superposed on the amplitude and phase transfer functions of the Fresnel reflection coefficient $\left[R\left(\xi+\beta_{0}\right)\right]$ versus the $x$ wavenumber $\xi+\beta_{0}$, here $\beta_{0}=12.56 \mu \mathrm{m}^{-1}$ The $\xi>0$ part of the spectrum is internally reflected $(|R|=1)$ but experiences a negative phase modulation that increases with $\xi$ approximately as $\sqrt{\xi}$ [lower dashed curve in Fig. 5(c)]. This varying phase causes a small positive shift to the reflected beam, which is well known as the Goos-Hänchen shift [27]. Because of the square root dependence of the phase near the critical wavenumber and the
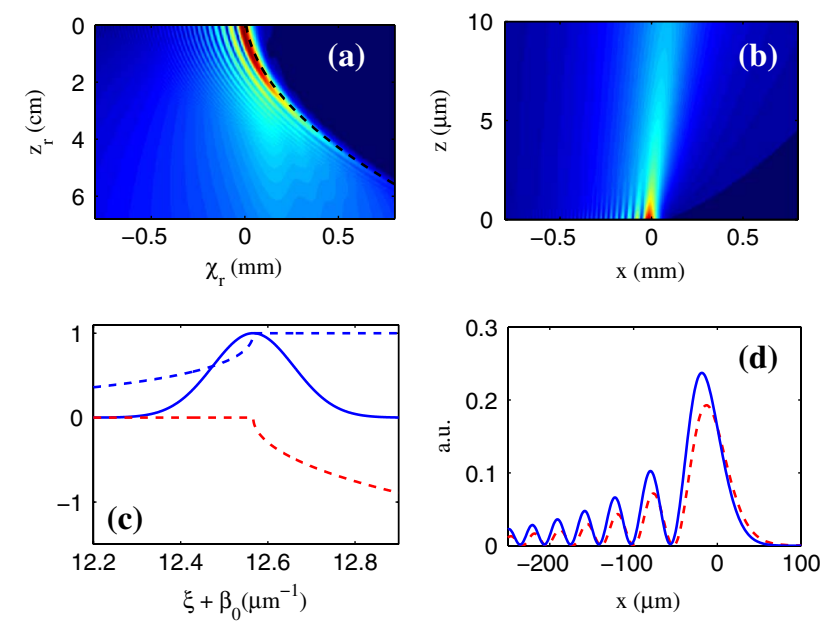

Fig. 5. (Color online) (a) Reflected and (b) transmitted wave amplitudes for the Airy beam of Fig. 2, impinging at angle $\theta_{1}=\theta_{c}=41.81^{\circ}$. (c) The Gaussian amplitude of the incident beam's spectrum in comparison with the amplitude (upper dashed curve) and phase (lower dashed curve) of the Fresnel reflection coefficient. For the phase curve, the ordinate is in radians, while for the amplitude curves, it is in arbitrary units (a.u.). (d) Intensity of the incident (solid) and reflected (dashed) beams at the interface, giving evidence to the Goos-Hänchen shift. 
relatively wide spectrum of the Airy beam, the Goos-Hänchen shift cannot be determined analytically and one resorts to directly comparing the amplitude profiles of the incident and reflected beams on the interface, as shown in Fig. 5(d). From the two maxima, the Goos-Hänchen shift is calculated to be $5.6 \mu \mathrm{m}$ or approximately 11 wavelengths.

In the previous TIR case, no caustic is created inside medium 2. This is because all rays that form the caustic, i.e., the rays corresponding to the positive part of the spectrum, impinge on the interface with angles slightly above $\theta_{1}=\theta_{c}$ and hence suffer TIR. It is however interesting to see what happens when some of the caustic-forming rays, or even all of them, escape TIR and are refracted through the interface. For example, in Fig. 6(a), the angle of incidence is slightly decreased to $\theta_{1}=\theta_{c}-0.5^{\circ}$. Now a part of the positive spectrum escapes TIR. The corresponding rays are refracted at grazing angles inside medium $2\left(\theta_{2}\right.$ below but very close to $\left.90^{\circ}\right)$, where they form a grazing caustic with acceleration toward the interface. Another interesting case is when $\theta_{1}=\theta_{c}$ and the incident Airy beam has its lobes developing toward positive $x$, i.e., when the negative sign is chosen in Eq. (14). Then the spectrum is as shown in Fig. 5(c), but now it is the $\xi<0$ part (or part below $\beta_{0}$ ) that forms the caustic and this part is partially transmitted $(|R|<1)$. As shown in Fig. $\underline{6(\mathrm{~b})}$, a clear grazing caustic is now formed with opposite sign of acceleration, i.e., bending away from the interface.

As previously discussed, Airy beams have been found to retain their diffraction-resisting and accelerating qualities within several adverse scenarios. It is thus here also interesting to investigate their behavior in relation with the TIR phenomenon. As shown from previous examples, however, e.g., Fig. 5(a), a single TIR has little effect on the reflected beam apart from the small Goos-Hänchen shift. In order to exaggerate the effect of TIR and assess the resulting performance of the Airy beam, we consider the scenario where an Airy beam is trapped and propagates through multiple, say $N$, TIRs inside a thin dielectric layer before it finally escapes and freely propagates in air [Fig. 7(a)]. Such configurations have been employed in experiments in order to make the Goos-Hänchen shift detectable [28]. Referring to Fig. 7(a), the wave amplitude, after the beam exits the dielectric slab and propagates
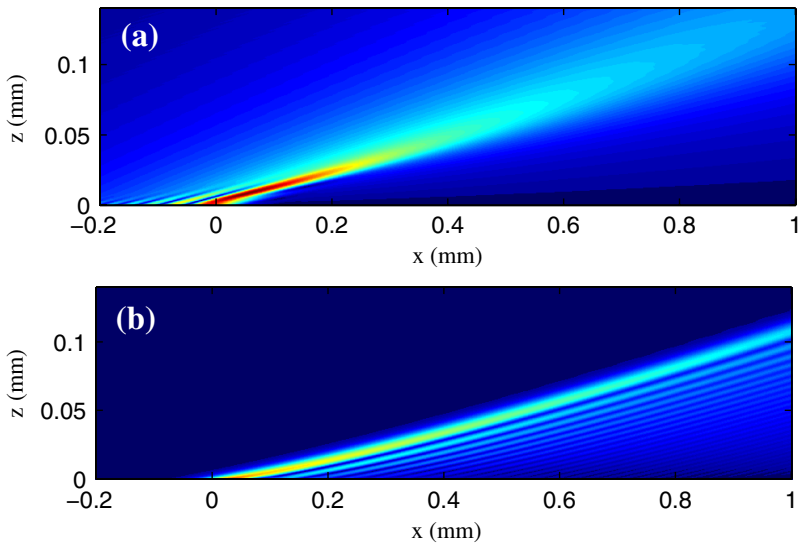

Fig. 6. (Color online) Transmitted wave amplitudes for the (a) Airy beam of Fig. 2, impinging at an angle $\theta_{1}=\theta_{c}-0.5^{\circ}=41.31^{\circ}$ and (b) Airy beam of Fig. 2 with reversed direction of lobes [negative sign in Eq. (14)] impinging at an angle $\theta_{1}=\theta_{c}=41.81^{\circ}$. freely in the medium $k_{2}$, is expressed in coordinates $\left(x_{b}, z_{b}\right)$ aligned to its axis as

$$
\begin{aligned}
u_{r}\left(x_{b}, z_{b}\right)= & \frac{1}{2 \pi} \int_{-\infty}^{+\infty} U_{0}(\xi) R^{N}\left(\beta_{0}+\xi \cos \theta_{1}\right) \tilde{T}(\xi) \\
& \times \exp \left(i \xi x_{b}-i \frac{\xi^{2}(N+1) h}{2 q_{10}}-i \frac{\xi^{2} z_{b}}{2 k_{2}}\right) \mathrm{d} \xi
\end{aligned}
$$

where $\tilde{T}$ is the Fresnel transmission coefficient for the final transition from the glass (medium $k_{1}$ ) to the air. The last equation assumes that the beam is launched exactly at the lower interface of the dielectric layer; that is why $(N+1)$ enters the equation. Propagation inside the input and output prisms is of little importance and is ignored. Also, the width $h$ of the layer should be small compared to the beam's diffraction length so that the effect of TIRs is isolated from mere diffraction. Note that, after the field envelope $u_{r}$ is computed, the actual field is obtained by an equation similar to Eq. (7), with the phase accordingly modified, namely with $s$ and $h$ replaced by $(N+1) s$ and $(N+1) h$, respectively. Results are shown in Figs. 7(b), $7(\mathrm{c})$, and $7(\mathrm{~d})$ for the case of $N=5,15,35$ TIRs, respectively, $\overline{\text { and }}$ for a slab with index 1.5 and width $h=0.5 \mathrm{~mm}$. It is apparent that the accelerating nature of the exiting beam persists. However, due to the $N$ times larger phase shift experienced by the positive $(\xi>0)$ spectral components, the beam is strongly right shifted. As the phase shift behaves like $\sqrt{\xi}$, rays with smaller $\xi$ are shifted more to compared to rays with larger $\xi$, resulting in increased diffraction that is manifested by the evident lobes spreading radially from different parts of the caustic.

Let us now turn to the grazing-incidence problem of Eq. (13). The input condition is taken to be an Airy beam accelerating toward the interface $(z=0)$

$$
u(x=0, z)=A i\left(\frac{z+\ell}{x_{0}}\right) \exp \left(a \frac{z+\ell}{x_{0}}+i v \frac{z}{x_{0}}\right),
$$

where $x_{0}$ is a length scale, $a$ is the apodization parameter, $\ell$ is a lateral offset, and $v$ accounts for a possible initial tilt of the
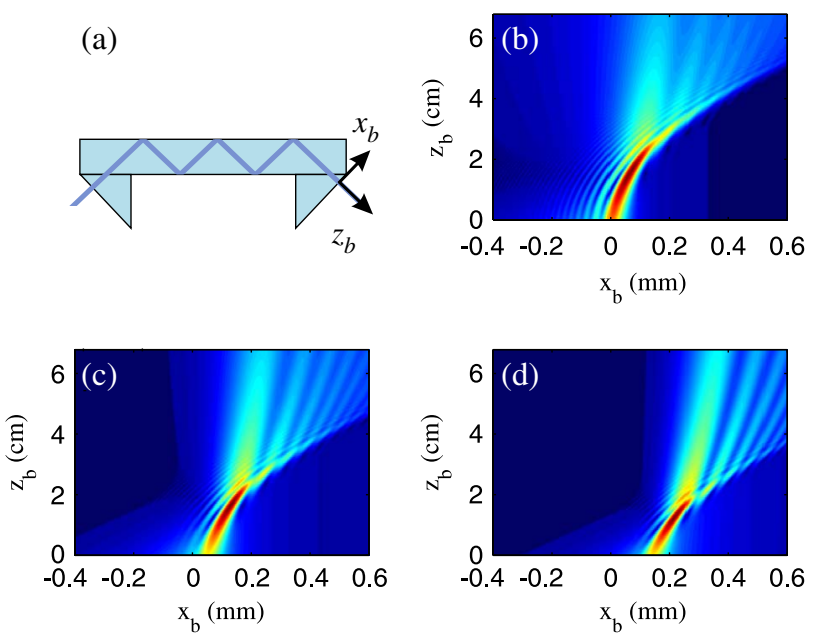

Fig. 7. (Color online) (a) Setting in which an optical beam undergoes multiple TIRs inside a dielectric layer of width $h$. (b)-(d) Amplitude of the exiting beam after (b) $N=5$, (c) $N=15$, and (d) $N=35$ TIRs. The index and width of the dielectric slab are taken as 1.5 and $0.5 \mathrm{~mm}$, respectively. 


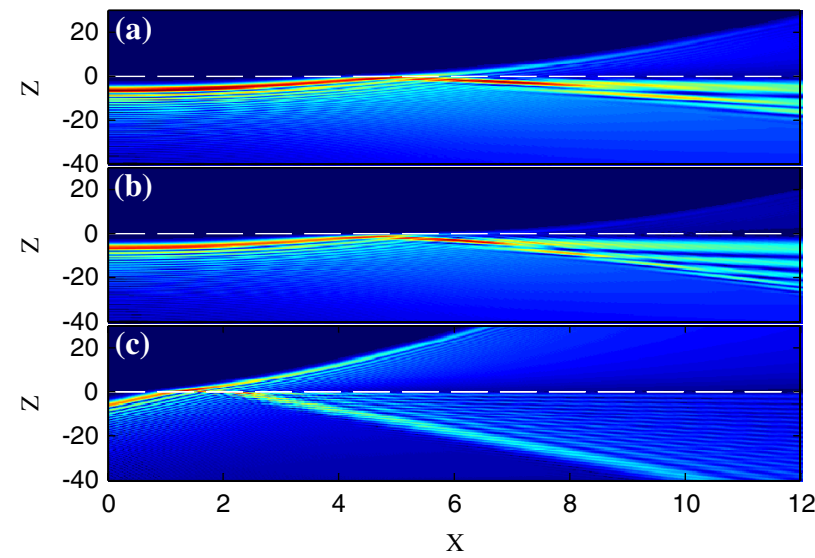

Fig. 8. (Color online) Grazing incidence of an Airy beam at the interface between two media with indices $n_{1}=1.001, n_{2}=1.0$. The beam parameters are $a=0.05, \ell=30 \mu \mathrm{m}$ and (a) $x_{0}=6 \mu \mathrm{m}, v=0$, (b) $x_{0}=10 \mu \mathrm{m}, v=0$, (c) $x_{0}=10 \mu \mathrm{m}, v=-5$. The vacuum wavelength is $\lambda=500 \mathrm{~nm}$.

beam. For paraxial beams, it is convenient to observe the propagation dynamics in scaled coordinates $Z=z / x_{0}, X=$ $x / k_{1} x_{0}^{2}$. Then the square root of the normalized potential barrier, i.e., $\left(k_{1}^{2}-k_{2}^{2}\right)^{1 / 2} x_{0} \equiv V_{0}^{1 / 2}$ is equal to the critical slope for TIR (with respect to the $Z$ axis) in scaled coordinates $(X, Z)$. For the Airy input wavefront of Eq. (19), the constituent rays propagate with slope $|Z+L|^{1 / 2}[5]$; hence, $Z_{c}=-L-V_{0}$, with $L=\ell / x_{0}$, is the exit position on plane $X=0$ of the first ray that escapes TIR.

Figure 8 (a) shows the amplitude evolution for the beam parameters $a=0.05, x_{0}=6 \mu \mathrm{m}$, and $\ell=30 \mu \mathrm{m}$ The fixed parameters are chosen $\lambda=500 \mathrm{~nm}, n_{1}=1.001, n_{2}=1.0$. The results were obtained by solving Eq. (13) through a splitstep Fourier method [29]. In this case, the normalized potential barrier is $V_{0} \cong 11$; therefore, rays starting from points $Z<$ $Z_{c}=-16$ on plane $X=0$ escape TIR and form the clear refracted caustic seen in medium 2. By increasing the transverse scale of the beam $x_{0}$, the potential barrier increases and more rays are trapped by TIR. This is shown in Fig. $\underline{8(\mathrm{~b})}$, where $x_{0}=$ $10 \mu \mathrm{m}\left(V_{0} \cong 32\right)$ and the beam is almost completely reflected. Imparting to the beam an input tilt toward the interface $(v<0)$, the potential barrier is lowered and the critical slope for TIR reduces to $V_{0}^{1 / 2}-|v|$. An example is shown in Fig. 8(c), where the beam of Fig. 8(b) has an initial tilt $v=-5$ (approximately $2.2^{\circ}$ ), allowing almost all inward rays to escape TIR and form a refracted caustic.

In the examples of Fig. 8 , it is also interesting to notice the beam amplitude in medium 1 . Contrary to the cases of Figs. 2-5, where the incident and reflected beam axes are well separated and a clear Airy-like reflected beam is observed, here the total observed field in medium 1 is the result of extended interference between the incident and reflected beams, which creates a modulated far-field pattern. For large enough potential barriers [e.g., Fig. 8(b)], the result is similar to having a zero Dirichlet boundary condition on the interface or, equivalently, the interference of two mirror Airy beams with phase difference $\pi$. Such far-field patterns are also typical in abruptly autofocusing waves as a result of interference of a continuum of Airy beams [22].

We finish by briefly investigating the incidence of an Airy beam on an interface to a LHM. In a medium of negative refractive index, an Airy beam retains its diffraction-resisting

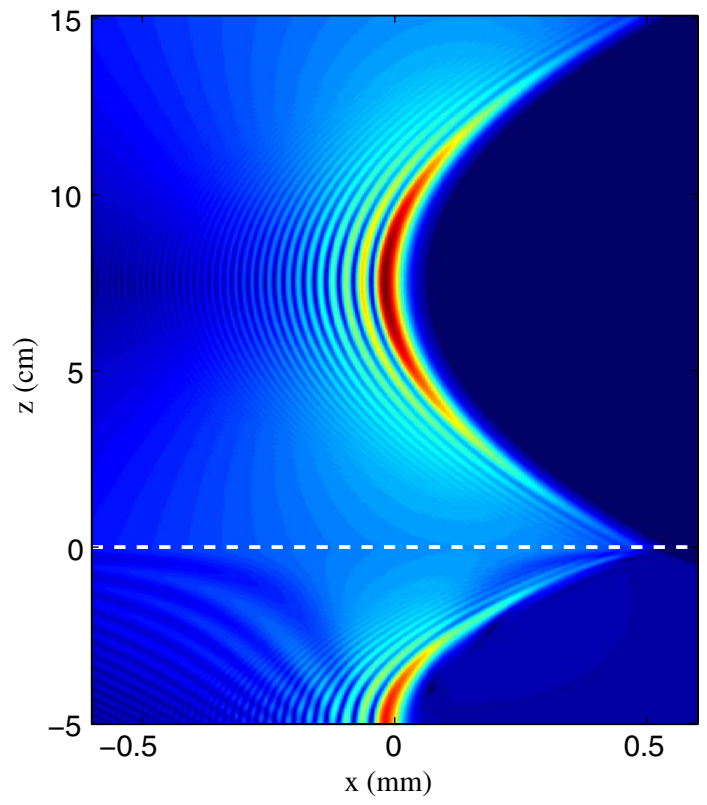

Fig. 9. (Color online) Normal incidence of the Airy beam of Fig. 2 propagating in air $\left(n_{1}=1\right)$ on the interface (white dashed line) to a LHM with refractive index $n_{2}=-1.5$. The beam is nearly perfectly imaged at the height of $7.5 \mathrm{~cm}$ inside the LHM. In medium 1, the total field (incident + reflected) is depicted.

and accelerating properties; however, the phase of the wave advances in an opposite direction to the power flow. This results in the inversion of Snell's law of refraction, in the sense that the beam is refracted at a negative angle $\theta_{2}$, but also in an inverse diffraction process that tends to cancel the diffraction that the beam has accumulated inside medium 1 . To see this, one notices that the amplitude of the beam transmitted in the LHM is given by Eqs. (9), (10), however, with $k_{2}=-\left|k_{2}\right|$ and $q_{20}=-\left(k_{2}^{2}-\beta_{0}^{2}\right)^{1 / 2}$, The $x$-wavenumber in medium 2 remains the same $\left(\xi+\beta_{0}\right)$ as dictated by the boundary condition at the interface. The Fresnel coefficients remain also the same since now both $\varepsilon_{2}$ and $\mu_{2}$ are negative. Now since $q_{20}<0$ in Eq. (10), the quadratic phase that causes the beam to spread initially decreases in magnitude. At $Z=-k_{1}^{2} q_{20}^{3} h /\left(k_{2}^{2} q_{10}^{3}\right)$, it is exactly zero and a nearly perfect image of the input beam is formed since the spectrum modulation induced by the Fresnel transmission coefficient is very weak $\left[T\left(\beta_{0}+\xi\right) \approx c t\right]$. Thereafter, the quadratic phase increases again and the beam diffracts. Note that distance $Z$ denotes the point on the refracted beam axis at which paraxial rays converge inside the LHM, a property that has been utilized to design perfect lenses [30]. An example is shown in Fig. 9 where the Airy beam of previous examples propagates in air for 10 diffraction lengths ( $h=10 L_{d 1} \cong 5 \mathrm{~cm}$ ) and impinges normally on the interface to a LHM with refractive index $n_{2}=-1.5$. Notice how the diffraction process is reversed inside the LHM and the beam gradually reconstructs until it creates a nearly perfect image of its input wavefront at height $Z=\left(n_{2} / n_{1}\right) h \cong 7.5 \mathrm{~cm}$.

\section{CONCLUSIONS}

We have studied analytically and numerically the reflection and refraction phenomena of a finite-power Airy beam at the interface between two dielectric half-spaces. Away from critical angles, the reflected and refracted beams retain their 
accelerating and diffraction-resisting properties, appearing as parabolic caustics, whose expressions were determined analytically. At Brewster's angle, the $p$-polarized reflected beam exhibits remarkable self-healing properties despite the severe filtering of its spectrum by the Fresnel coefficient. Incidence near but not exactly at Brewster's angle was also examined. In the other interesting case, namely the TIR regime, we found that escaping rays can create clear and grazing to the interface caustics. We also observed the Goos-Hänchen effect, which however cannot be computed analytically for an Airy beam. In order to further investigate the TIR phenomenon, we also investigated Airy beams undergoing multiple TIRs inside a dielectric film. We found that the accelerating character of the exiting beam persists, although at the cost of a large Goos-Hänchen shift and strong diffraction. We also considered TIR at the grazing incidence to an interface between two media with close refractive indices and reverse diffraction and perfect imaging of an Airy beam inside a LHM.

Airy beams are currently becoming increasingly useful for delivering optical power and manipulating particles in various optical settings. The analytical and numerical developments of the present work will be useful and provide insight in configurations where these versatile structured optical waves encounter interfaces or propagate inside layered media.

\section{ACKNOWLEDGMENTS}

The research leading to these results has received funding from the European Union's Seventh Framework Programme (FP7-REGPOT-2009-1) under grant agreement no. 245749.

\section{REFERENCES}

1. M. Berry and N. Balazs, "Non-spreading wave packets," Am. J. Phys. 47, 264-267 (1979).

2. G. Siviloglou and D. Christodoulides, "Accelerating finite energy Airy beams," Opt. Lett. 32, 979-981 (2007).

3. G. A. Siviloglou, J. Broky, A. Dogariu, and D. N. Christodoulides, "Observation of accelerating Airy beams," Phys. Rev. Lett. 99, 213901 (2007)

4. G. A. Siviloglou, J. Broky, A. Dogariu, and D. N. Christodoulides, "Ballistic dynamics of Airy beams," Opt. Lett. 33, 207-209 (2008).

5. Y. Kaganovsky and E. Heyman, "Wave analysis of Airy beams," Opt. Express 18, 8440-8452 (2010).

6. J. Durnin, J. Miceli, and J. Eberly, "Diffraction-free beams," Phys. Rev. Lett. 58, 1499-1501 (1987).

7. J. Gutiérrez-Vega, M. Iturbe-Castillo, and S. Chávez-Cerda, "Alternative formulation for invariant optical fields: Mathieu beams," Opt. Lett. 25, 1493-1495 (2000).

8. M. Bandres, J. Gutiérrez-Vega, and S. Chávez-Cerda, "Parabolic nondiffracting optical wave fields," Opt. Lett. 29, 44-46 (2004).
9. I. Besieris and A. Shaarawi, "A note on an accelerating finite energy Airy beam," Opt. Lett. 32, 2447-2449 (2007).

10. J. Broky, G. Siviloglou, A. Dogariu, and D. N. Christodoulides, "Self-healing properties of optical Airy beams," Opt. Express 16, 12880-12891 (2008).

11. X. Chu, "Evolution of an Airy beam in turbulence," Opt. Lett. 36, 2701-2703 (2011).

12. Y. Hu, S. Huang, P. Zhang, C. Lou, J. Xu, and Z. Chen, "Persistence and breakdown of Airy beams driven by an initial nonlinearity," Opt. Lett. 35, 3952-3954 (2010).

13. Y. Hu, P. Zhang, C. Lou, S. Huang, J. Xu, and Z. Chen, "Optimal control of the ballistic motion of Airy beams," Opt. Lett. 35, 2260-2262 (2010).

14. J. Baumgartl, M. Mazilu, and K. Dholakia, "Optically mediated particle clearing using Airy wavepackets," Nat. Photon. 2, 675-678 (2008)

15. D. Christodoulides, "Optical trapping: riding along an Airy beam," Nat. Photon. 2, 652-653 (2008).

16. P. Zhang, J. Prakash, Z. Zhang, M. S. Mills, N. K. Efremidis, D. N. Christodoulides, and Z. Chen, "Trapping and guiding microparticles with morphing autofocusing Airy beams," Opt. Lett. 36, 2883-2885 (2011).

17. J.-X. Li, W.-P. Zang, and J.-G. Tian, "Vacuum laser-driven acceleration by Airy beams," Opt. Express 18, 7300-7306 (2010).

18. P. Polynkin, M. Kolesik, J. Moloney, G. Siviloglou, and D. Christodoulides, "Curved plasma channel generation using ultraintense Airy beams," Science 324, 229-232 (2009).

19. A. Salandrino and D. N. Christodoulides, "Airy plasmon: a nondiffracting surface wave," Opt. Lett. 35, 2082-2084 (2010).

20. P. Zhang, S. Wang, Y. Liu, X. Yin, C. Lu, Z. Chen, and X. Zhang, "Plasmonic Airy beams with dynamically controlled trajectories," Opt. Lett. 36, 3191-3193 (2011).

21. A. Minovich, A. E. Klein, N. Janunts, T. Pertsch, D. N. Neshev, and Y. S. Kivshar, "Generation and near-field imaging of Airy surface plasmons," Phys. Rev. Lett. 107, 116802 (2011).

22. N. K. Efremidis and D. N. Christodoulides, "Abruptly autofocusing waves," Opt. Lett. 35, 4045-4047 (2010).

23. I. M. Besieris and A. M. Shaarawi, "Accelerating Airy wave packets in the presence of quadratic and cubic dispersion," Phys. Rev. E 78, 046605 (2008).

24. A. Chong, W. H. Renninger, D. N. Christodoulides, and F. W. Wise, "Airy-Bessel wave packets as versatile linear light bullets,” Nat. Photon. 4, 103-106 (2010).

25. Y. Fattal, A. Rudnick, and D. M. Marom, "Soliton shedding from Airy pulses in Kerr media," Opt. Express 19, 17298-17307 (2011).

26. C. Ament, P. Polynkin, and J. V. Moloney, "Supercontinuum generation with self-healing Airy pulses," in CLEO 2011-Laser Applications to Photonic Applications, OSA Technical Digest (CD) (Optical Society of America, 2011), paper PDPC9.

27. F. Goos and H. Hänchen, "Ein neuer und fundamentaler Versuch zur Totalreflexion," Ann. Phys. 436, 333-346 (1947).

28. M. Green, P. Kirkby, and R. S. Timsit, "Experimental results on the longitudinal displacement of light beams near total reflection," Phys. Lett. A 45, 259-260 (1973).

29. G. Agrawal, Nonlinear Fiber Optics, 3rd ed. (Academic 2001)

30. J. B. Pendry, "Negative refraction makes a perfect lens," Phys. Rev. Lett. 85, 3966-3969 (2000). 